\title{
All, some or none? Statin prescribing for frail older adults
}

\author{
Sudeep S. Gill MD MSc
}

- Cite as: CMAJ 2019 January 14;191:E30-1. doi: 10.1503/cmaj.181671

See related article at www.cmaj.ca/lookup/doi/10.1503/cmaj.180853

$\mathbf{T}$ he discovery of statins was built on decades of scientific insights, ${ }^{1}$ and randomized controlled trials (RCTs) conducted over the past 25 years have established their benefits for managing cardiovascular disease in various patient populations. ${ }^{2}$ The use of statins has likely contributed to observed reductions in mortality from cardiovascular disease. ${ }^{3}$

Several important debates have influenced prescribing practices with regard to statins. ${ }^{2,4}$ In a linked research article, Campitelli and colleagues address one such debate involving the merits of more versus less intensive statin dosing in a vulnerable population that has not yet been well represented in RCTs. ${ }^{5}$

A widely cited 2010 meta-analysis of 5 RCTs (which included 39612 participants) found more intensive statin regimens reduced major vascular events compared with less intensive regimens (rate ratio $0.85,95 \%$ confidence interval $[\mathrm{Cl}] 0.82$ to 0.89 ). ${ }^{6}$ However, frail older adults were mostly ineligible to enroll in these RCTs. ${ }^{7}$ As a result, questions remain about the generalizability of the findings of the meta-analysis and the balance of benefits and risks of intensive statin regimens in vulnerable populations.

One vulnerable population commonly prescribed statins is the large and growing group of frail older adults who reside in long-term care facilities. Despite the high prevalence of cardiovascular disease in these individuals, they are routinely excluded from the RCTs that influence the clinical practice guidelines that, in turn, influence their medication prescriptions.?

Campitelli and colleagues confront this knowledge gap in a carefully planned retrospective cohort study that used propensityscore matching to compare outcomes in long-term care residents who were prevalent users of either intensive-dose or moderate-dose statins; ${ }^{5}$ the authors look at several outcomes including all-cause mortality and admissions to hospital for cardiovascular events. They identified 21808 participants who were taking statins, of whom $4762(21.8 \%)$ received intensive-dose statins and $17046(78.2 \%)$ received moderate-dose statins. They were able to match 4577 pairs of participants taking intensiveand moderate-doses of statins. After 1 year, more than a quarter had died and more than $10 \%$ had been admitted to hospital for cardiovascular conditions. Compared with use of moderate-dose statins, there was no significant association between use of

\section{KEY POINTS}

- Treatment with statins has become a fundamental component of the management of cardiovascular disease for many patients.

- Evidence from randomized controlled trials (RCTs) suggests that use of intensive-dose statins may reduce cardiovascular events compared with moderate-dose statins; however, these findings have limited generalizability as frail older adults were underrepresented in RCTs.

- Intensive-dose statin regimens may not confer benefits for older residents of long-term care facilities.

- Clinicians should tailor statin therapy (intensive dose v. moderate dose or any dose v. none) to the individual older adult's prognosis and preferences.

intensive-dose statins and either overall survival (hazard ratio [HR] $0.97,95 \% \mathrm{Cl} 0.90$ to 1.05 ) or admission to hospital for cardiovascular events ( $\mathrm{HR} 0.99,95 \% \mathrm{Cl} 0.88$ to 1.12 ). ${ }^{5}$

It is worth noting that study participants were, on average, roughly 2 decades older than participants in the RCTs that evaluated more versus less intensive use of statins. ${ }^{6}$ Although few of the long-term care residents would have been eligible for entry into these RCTs, more than 1 in 5 of the study cohort were prescribed intensive-dose statins. Study participants also bore a high burden of functional dependence, comorbid disease and medication use. For example, only 1 in 10 participants was independent in performing activities of daily living. More than a third of the cohort had diabetes, and more than half were diagnosed with Alzheimer disease or another dementia. One year after entry into the study cohort, $25 \%$ of the cohort had died.

The results of Campitelli and colleagues' main analysis were consistent in stratified analyses, including one defined by frailty status. However, the authors were unable to examine statinassociated adverse events, likely a wise omission given the study's prevalent user design and the ongoing debate about the range of specific adverse events that can be attributed to statin exposure. ${ }^{2,4}$

Campitelli and colleagues have usefully spotlighted an often neglected, yet growing, population of frail older adults who reside 
in long-term care facilities. ${ }^{8}$ Like many similar nations, Canada is experiencing a dramatic demographic shift. Since 2016, there are now more people aged 65 years and older in Canada than there are children aged 14 years or younger. ${ }^{8}$ It is projected that the number of Canadians aged 65 years and older will continue to rise and, by 2024 , they will account for over $20 \%$ of the population. Although increased life expectancy should be celebrated as a product of successful breakthroughs - which includes the use of statins to manage cardiovascular disease - Canada's health care system has not yet adapted to the care needs of its growing population of older adults, many of whom will likely develop chronic diseases that will require complex care.

Another vulnerable population that has not been well represented in RCTs of statins until recently is individuals approaching the end of life, which overlaps somewhat with the population of older adults in long-term care. Patients in palliative care settings, however, are more likely to have rapidly progressive conditions such as cancers. Fewer than 1 in 10 participants in Campitelli and colleagues' study had a diagnosis of cancer. Research has shown that statins continue to be prescribed to many patients with limited life expectancy, including patients with cancer who filled statin prescriptions within 30 days of death. ${ }^{9}$ To address this situation, a novel study randomly assigned individuals with an estimated life expectancy of between 1 month and 1 year and no recent active cardiovascular disease to either stop or continue their statin therapy. ${ }^{10}$ Just under half of the participants had cancer. The investigators found no significant difference in 60-day mortality ( $23.8 \%$ v. $20.3 \%, 90 \% \mathrm{Cl}-3.5 \%$ to $10.5 \%$ ), better quality of life and a reduction in medication costs with statin deprescribing. This RCT provides evidence to help guide clinicians' decisions about whether or not to reduce medication burden in patients with advanced disease and limited life expectancy.

When contemplating whether prescribing intensive-dose statins would benefit older adults who are frail, physicians should perform an individualized assessment and incorporate patients' preferences whenever possible. Some patients may prefer to start or continue intensive-dose statins based on their assessment of the potential benefits and risks of this treatment approach. A growing literature base now supports alternative approaches, including "some" (i.e., moderate-dose statins, which are supported by the findings of the linked study) and "none" (in line with the deprescribing approach examined in other recent work). ${ }^{9,10}$

\section{References}

1. Endo A. A historical perspective on the discovery of statins. Proc Jpn Acad Ser B Phys Biol Sci 2010;86:484-93.

2. Collins R, Reith C, Emberson J, et al. Interpretation of the evidence for the efficacy and safety of statin therapy. Lancet 2016;388:2532-61.

3. Tu JV, Nardi L, Fang J, et al.; Canadian Cardiovascular Outcomes Research Team. National trends in rates of death and hospital admissions related to acute myocardial infarction, heart failure and stroke, 1994-2004. CMAJ 2009; 180:E118-25.

4. Brown C. Statin-use debate creates furor at The BMJ. CMAJ 2014;186:E405-6.

5. Campitelli MA, Maxwell CJ, Maclagan LC, et al. One-year survival and admission to hospital for cardiovascular events among older residents of long-term care facilities who were prescribed intensive- and moderate-dose statins. CMAJ 2019;191:E32-9.

6. Cholesterol Treatment Trialists' (CTT) Collaboration; Baigent C, Blackwell L, Emberson J, et al. Efficacy and safety of more intensive lowering of LDL cholesterol: a meta-analysis of data from 170000 participants in 26 randomised trials. Lancet 2010;376:1670-81.

7. Bourgeois FT, Orenstein L, Ballakur S, et al. Exclusion of elderly people from randomized clinical trials of drugs for ischemic heart disease. J Am Geriatr Soc 2017;65:2354-61.

8. The State of Seniors Health Care in Canada. Ottawa: Canadian Medical Association; 2016. Available: www.cma.ca/En/Lists/Medias/the-state-of-seniors-health-care-in -canada-september-2016.pdf (accessed 2018 Dec. 18).

9. Holmes HM, Todd A. Evidence-based deprescribing of statins in patients with advanced illness. JAMA Intern Med 2015;175:701-2.

10. Kutner JS, Blatchford PJ, Taylor DH, et al. Safety and benefit of discontinuing statin therapy in the setting of advanced, life-limiting illness: a randomized clinical trial. JAMA Intern Med 2015;175:691-700.

Competing interests: Sudeep Gill has collaborated with Campitelli and colleagues on unrelated work. No other competing interests were declared.

This article was solicited and has not been peer reviewed.

Affiliation: Department of Medicine, Queen's University, Kingston, Ont.

Correspondence to: Sudeep Gill, ssg@queensu.ca 